\title{
Modelling of Reactive and Non-Reactive Multiphase Flows
}

\author{
Markus Klein ${ }^{1, *(\mathbb{D})}$ and Nilanjan Chakraborty ${ }^{2}$ (D) \\ 1 Department of Aerospace Engineering, Bundeswehr University Munich, 85577 Neubiberg, Germany \\ 2 School of Engineering, Newcastle University, Newcastle upon Tyne NE1 7RU, UK; \\ nilanjan.chakraborty@newcastle.ac.uk \\ * Correspondence: markus.klein@unibw.de; Tel.: +49-(0)89-6004-2122
}

Citation: Klein, M.; Chakraborty, N. Modelling of Reactive and

Non-Reactive Multiphase Flows. Fluids 2021, 6, 304. https://doi.org/ 10.3390/fluids6090304

Received: 18 August 2021

Accepted: 20 August 2021

Published: 27 August 2021

Publisher's Note: MDPI stays neutral with regard to jurisdictional claims in published maps and institutional affiliations.

Copyright: (C) 2021 by the authors Licensee MDPI, Basel, Switzerland. This article is an open access article distributed under the terms and conditions of the Creative Commons Attribution (CC BY) license (https:// creativecommons.org/licenses/by/ $4.0 /)$.
Multiphase flows are found in several industrial processes encompassing power generation, pharmaceutical and chemical industry and agriculture. The production of chemical goods worth billions of dollars and the generation of several hundred trillion Joules of primary energy depend on the safe and efficient handling of multiphase flows. Hence, their control and accurate numerical prediction are of paramount importance for the development of future-generation high-efficiency cost-effective engineering devices.

Key challenges associated with the modelling of such flows include their multiphysics and multiscale nature involving interactions of turbulence, interface physics, phase change and chemical reactions on temporal and spatial scales spanning several orders of magnitude. In the last two decades, progress in numerical methods and computing power allowed the impressive direct numerical simulations (DNS) of multiphase flows which considerably improved our physical understanding of such flows. However, as DNS are limited to academic configurations in the foreseeable future, the development of next-generation models for large-scale or averaged multiphase flows is an important challenge.

The Special Issue "Modelling of Reactive and Non-Reactive Multiphase Flows" collects 11 papers covering a broad range of topics dealing with applications of non-reactive [1-6] and reactive [7-11] multiphase flows in natural environment as well as technical applications. The contributions address important numerical and modelling issues, which not only cover fundamental physics but also address the aspect of application.

The characterization of the fall speeds of raindrops is the main topic in Ren et al. [1]. For the first time, computations of droplets in turbulent surroundings are conducted with a DNS code based on a volume of the fluid method, and results for the drop surface deformation and its internal circulation are reported. It has been found that the terminal velocity decreases in turbulent flows which has been explained by a shortening of the wake recirculation region. Further, the turbulent surroundings trigger substantial rises in the drop axis ratio amplitude and a slight increase in the drop oscillation frequency.

Although many processes of practical interest can be considered as two-phase flows with Newtonian behavior in both phases, there are several relevant applications where the continuous liquid phase exhibits non-Newtonian flow characteristics. Its influence on the flow statistics of a turbulent bubble-laden downflow in a vertical channel was investigated in Bräuer et al. [2], where a DNS study was conducted for power-law fluids with a range of power-law indexes. The resulting first- and second-order fluid statistics, i.e., the gas fraction, mean velocity and velocity fluctuation profiles across the channel, show clear trends in reply to varying power-law indexes. In addition, it was observed that bubble oscillations increase with decreasing power-law index.

Horizontal two-phase flows are common in nature, such as the movement of water droplets in the air or the formation of waves on water surfaces, but they also occur in industrial processes, for example, in wave-like flows in pressurized water reactors. Their modelling and experimental characterization have been addressed by Höhne et al. [3] and Carraretto et al. [4]. The modelling [3] has been performed within the two-fluid Euler-Euler approach. A modified formulation of the morphology detection functions 
within the algebraic interfacial area density model has been presented in combination with different models for the drag force acting on a sheared gas-liquid interface. The transient simulations of the WENKA counter-current stratified two-phase flow experiment were performed for validation. The prediction of the correct flow pattern as observed in the experiment improved dramatically, when a turbulence damping term was included in the standard two-equation models. The experimental characterization of stratified air-water flows is presented in [4]. The pressure gradient along the pipeline was determined together with the void fraction. None of the existing models is able to predict both quantities throughout the range of operating conditions. Eventually, with both the measured pressure gradient and the void fraction, a two-fluid model was implemented in order to determine the interfacial shear stress and to compare the outcome with the literature.

Hydrodynamic cavitation occurs in many engineering applications, ranging from fast-running ship propellers to artificial heart valves, where it is associated with detrimental effects. However, acoustic cavitation was found to be useful for ultrasonic cleaning, for medical imaging or in the context of micropumps [5]. To study the physics in detail, a fully coupled pressure-based algorithm and finite-volume framework for the simulation of the acoustic cavitation of bubbles in polytropic gas-liquid systems has been proposed and extensively validated by Denner et al. [5].

The description of a sharp interface with its associated numerical difficulties is common to many two-phase flow simulation problems. A new compressible framework within OpenFOAM, which incorporates mitigation strategies for the well-known issue of spurious currents, has been presented by Tretola and Vogiatzaki [6]. The framework incorporates the compressible algebraic volume of fluid method with additional interfacial treatment techniques including volume fraction smoothing and sharpening (for the calculation of the interface geometries and surface tension force, respectively) as well as the filtering of capillary forces and has been tested against different benchmarks.

The work of Gärtner et al. [7] represents the transition from non-reactive to reactive multiphase flows. During certain operating conditions in spark-ignited direct injection engines, the injected fuel is superheated and begins to rapidly vaporize. Fast vaporization can be beneficial for fuel-oxidizer mixing and subsequent combustion, but it poses the risk of spray collapse, which has been numerically investigated for a single-hole injector and an eight-hole injector. The numerical results match well with experimental data in terms of the spray structures and support the assumption that the interaction of shocks due to the under expanded vapor jet causes spray collapse.

Fundamental aspects of spray flame interaction have been analysed by Ozel-Erol and Chakraborty [8]. Three-dimensional carrier phase DNS of V-shaped n-heptane spray flames have been performed for different initially mono-sized droplet diameters to investigate the influence of the mean flow velocity on the burning rate and the flame structure. The following conclusions have been drawn: (1) the extent of flame wrinkling for droplet cases is greater than that of the corresponding gaseous premixed flames due to the flame-droplet interaction; (2) the major part of the heat release arises due to the premixed mode of combustion; and (3) the mean value of consumption speed diminishes with increasing droplet diameters. The residence time of the droplets within the flame decreases with increasing mean inflow velocity, and the droplets can survive for larger axial distances before the completion of their evaporation, which leads to greater extents of the flamedroplet interaction.

Dressler et al. [9] studied the Sydney diluted spray burner by performing large eddy simulation in combination with the Eulerian stochastic fields (ESFs) method together with a tabulated chemistry approach. The spray is treated using a two-way coupled Eulerian-Lagrangian procedure. Comparisons with the artificially thickened flame (ATF) approach indicate that the ESF results better describe the flame entrainment into the cold spray core of the flame. Further, the dynamics of the subgrid scalar contributions were investigated, and the reconstructed probability density distributions were compared to common presumed shapes qualitatively and quantitatively in the context of spray 
combustion. It is demonstrated that the ESF method can be a valuable tool to evaluate approaches relying on the pre-integration of the thermochemical lookup table.

Emissions of $\mathrm{CO}_{2}$ as well as $\mathrm{NO}$ and soot during the combustion in power generation processes must be reduced to comply with stricter legal regulations, which requires the development of new combustion technologies such as the moderate or intense low-oxygen dilution (MILD) combustion [10]. To this end, the structures of both gaseous and liquid ethanol flames in different oxidizing gas environments in the axisymmetric counterflow configuration at atmospheric pressure were studied by Norena and Gutheil [10]. It was confirmed that ethanol spray flames in air show two reaction zones at low strain whereas the lean ethanol spray flames in pure oxygen exhibit a single-reaction zone in all situations studied. An analysis regarding the addition of $\mathrm{CO}_{2}$ in both the ethanol/oxygen gas and spray flames was also discussed, and it was found that the $\mathrm{CO}_{2}$ dilution of the carrier gas spray is much more efficient than diluting the opposed gas stream in the counterflow configuration for the generation of MILD combustion conditions in oxy-fuel flames.

Finally, the technology of particle synthesis in spray flames has been addressed by Wollny et al. [11]. Platinum-decorated alumina particles have the potential to be a highly effective catalyst. The particles are synthesized from platinum(II) acetylacetonate dissolved in a mixture of isopropanol and acetic acid with dispersed alumina carriers. The process is simulated by means of large eddy simulation with reaction kinetics and aerosol dynamics modeling. A two-mixture-fraction approach to tabulated chemistry with a thickened flame model was used to consider the complex reaction kinetics of the solvent spray combustion combined with an extended model for aerosol dynamics, capable of predicting the deposition rate and the surface particle growth. The ratio of deposited platinum on the alumina carrier particles and the mean diameters of the deposited particles are in good agreement with the experimental observation.

We are grateful to all authors and the reviewers for their valuable contributions. Finally, we would like to express our gratitude to the editorial team for providing us with the opportunity to write this Special Issue and all the help in the editorial process.

Author Contributions: Writing — original draft preparation, M.K.; writing—review and editing, N.C., M.K. Both authors have read and agreed to the published version of the manuscript.

Funding: This research received no external funding.

Institutional Review Board Statement: Not applicable.

Informed Consent Statement: Not applicable.

Data Availability Statement: Not applicable.

Conflicts of Interest: The authors declare no conflict of interest.

\section{References}

1. Ren, W.; Reutzsch, J.; Weigand, B. Direct Numerical Simulation of Water Droplets in Turbulent Flow. Fluids 2020, 5, 158. [CrossRef]

2. Bräuer, F.; Trautner, E.; Hasslberger, J.; Cifani, P.; Klein, M. Turbulent Bubble-Laden Channel Flow of Power-Law Fluids: A Direct Numerical Simulation Study. Fluids 2021, 6, 40. [CrossRef]

3. Höhne, T.; Porombka, P.; Moya Sáez, S. Validation of AIAD Sub-Models for Advanced Numerical Modelling of Horizontal Two-Phase Flows. Fluids 2020, 5, 102. [CrossRef]

4. Carraretto, I.M.; Colombo, L.P.M.; Fasani, D.; Guilizzoni, M.; Lucchini, A. Pressure Drop and Void Fraction in Horizontal Air-Water Stratified Flows with Smooth Interface at Atmospheric Pressure. Fluids 2020, 5, 101. [CrossRef]

5. Denner, F.; Evrard, F.; van Wachem, B. Modeling Acoustic Cavitation Using a Pressure-Based Algorithm for Polytropic Fluids. Fluids 2020, 5, 69. [CrossRef]

6. Tretola, G.; Vogiatzaki, K. Numerical Treatment of the Interface in Two Phase Flows Using a Compressible Framework in OpenFOAM: Demonstration on a High Velocity Droplet Impact Case. Fluids 2021, 6, 78. [CrossRef]

7. Gärtner, J.W.; Feng, Y.; Kronenburg, A.; Stein, O.T. Numerical Investigation of Spray Collapse in GDI with OpenFOAM. Fluids 2021, 6, 104. [CrossRef]

8. Ozel Erol, G.; Chakraborty, N. Effects of Mean Inflow Velocity and Droplet Diameter on the Propagation of Turbulent V-Shaped Flames in Droplet-Laden Mixtures. Fluids 2021, 6, 1. [CrossRef] 
9. Dressler, L.; Sacomano Filho, F.L.; Ries, F.; Nicolai, H.; Janicka, J.; Sadiki, A. Numerical Prediction of Turbulent Spray Flame Characteristics Using the Filtered Eulerian Stochastic Field Approach Coupled to Tabulated Chemistry. Fluids 2021, 6, 50. [CrossRef]

10. Noreña, O.; Gutheil, E. Structures of Ethanol Spray Flames under CO2 Dilution of the Oxidizer in the Counterflow Configuration under MILD Combustion Conditions. Fluids 2020, 5, 194. [CrossRef]

11. Wollny, P.; Angel, S.; Wiggers, H.; Kempf, A.M.; Wlokas, I. Multiscale Simulation of the Formation of Platinum-Particles on Alumina Nanoparticles in a Spray Flame Experiment. Fluids 2020, 5, 201. [CrossRef] 